\title{
Randomized phase II trial of uracil/tegafur and cisplatin versus pemetrexed and cisplatin with concurrent thoracic radiotherapy for locally advanced unresectable stage III non-squamous non-small cell lung cancer: NJLCG1001
}

\author{
Kana Watanabe ${ }^{1}$, Yukihiro Toi ${ }^{2}$, Atsushi Nakamura ${ }^{2}$, Ryosuke Chiba ${ }^{3}$, Masachika Akiyama ${ }^{3}$, \\ Jun Sakakibara-Konishi ${ }^{4}$, Hisashi Tanaka ${ }^{5}$, Naruo Yoshimura ${ }^{6}$, Eisaku Miyauchi ${ }^{7}$, Taku Nakagawa ${ }^{8}$, \\ Ryotaro Igusa $^{9}$, Hiroyuki Minemura ${ }^{10}$, Yoshiaki Mori ${ }^{11}$, Keisuke Fujimoto ${ }^{12}$, Haruo Matsushita ${ }^{13}$, \\ Fumiaki Takahashi $^{14}$, Tatsuro Fukuhara ${ }^{1}$, Akira Inoue ${ }^{15}$, Shunichi Sugawara ${ }^{2}$, Makoto Maemondo ${ }^{3}$; \\ North Japan Lung Cancer Study Group, Sendai, Japan
}

${ }^{1}$ Department of Respiratory Medicine, Miyagi Cancer Center, Natori, Japan; ${ }^{2}$ Department of Pulmonary Medicine, Sendai Kousei Hospital, Sendai, Japan; ${ }^{3}$ Division of Pulmonary Medicine, Department of Internal Medicine, Iwate Medical University School of Medicine, Yahaba, Japan; ${ }^{4}$ First Department of Medicine, Hokkaido University Hospital, Sapporo, Japan; ${ }^{5}$ Department of Respiratory Medicine, Hirosaki University, Hirosaki, Japan; ${ }^{6}$ Department of Respiratory Medicine, Tohoku Medical and Pharmaceutical University, Sendai, Japan; ${ }^{7}$ Department of Respiratory Medicine, Tohoku University School of Medicine, Sendai, Japan; ${ }^{8}$ Department of Thoracic Surgery, Omagari Kosei Medical Center, Daisen, Japan; ${ }^{9}$ Department of Respiratory Medicine, Osaki Citizen Hospital, Osaki, Japan; ${ }^{10}$ Department of Pulmonary Medicine, Fukushima Medical University School of Medicine, Fukushima, Japan; ${ }^{11}$ Department of Respiratory Medicine, Iwate Prefectural Central Hospital, Morioka, Japan; ${ }^{12}$ Department of Radiation Oncology, Miyagi Cancer Center, Natori, Japan; ${ }^{13}$ Department of Radiation Oncology, Tohoku University School of Medicine, Sendai, Japan; ${ }^{14}$ Department of Information Science, Iwate Medical University, Morioka, Japan; ${ }^{15}$ Department of Palliative Medicine, Tohoku University School of Medicine, Sendai, Japan

Contributions: (I) Conception and design: K Watanabe, S Sugawara, M Maemondo; (II) Administrative support: None; (III) Provision of study materials or patients: All authors; (IV) Collection and assembly of data: All authors; (V) Data analysis and interpretation: K Watanabe, M Maemondo, F Takahashi; (VI) Manuscript writing: All authors; (VII) Final approval of manuscript: All authors.

Correspondence to: Makoto Maemondo, MD, PhD. Division of Pulmonary Medicine, Department of Internal Medicine, Iwate Medical University School of Medicine, 2-1-1 Idaidori Yahaba-cho Shiwa-gun Iwate 028-3694, Japan. Email: maemondo-ma693@aioros.ocn.ne.jp.

Background: The optimal regimen for concurrent chemoradiotherapy (CCRT) of locally advanced nonsquamous non-small cell lung cancer (NSCLC) was not definitive. We conducted randomized phase II study, NJLCG0601, and chemoradiotherapy with uracil/tegafur (UFT) and cisplatin achieved promising efficacy without severe toxicities. Here, we evaluated between this regimen and pemetrexed plus cisplatin in chemoradiotherapy for stage III non-squamous NSCLC.

Methods: Patients with inoperable stage III non-squamous NSCLC were randomly assigned in a 1:1 ratio to UFT $400 \mathrm{mg} / \mathrm{m}^{2}$ on days $1-14$ and 29-42, and cisplatin $80 \mathrm{mg} / \mathrm{m}^{2}$ on days 8 and 36 (UP), or cisplatin $75 \mathrm{mg} / \mathrm{m}^{2}$ and pemetrexed $500 \mathrm{mg} / \mathrm{m}^{2}$ on days 1,22 , and 43 (PP). Involved-field radiotherapy (IFRT) underwent from day 1 to a total dose of 66 Gy in 33 fractions. Consolidation chemotherapy after CCRT was prohibited for this study. The primary endpoint was defined as 2-year overall survival (OS). This trial was registered in the University Hospital Medical Information Network Clinical Trials Registry (UMIN000003948). Results: From November 2010 to June 2017, 86 patients were entered from 11 institutions. Median followup was 54 months. Of the 85 eligible patients, the 2-year OS rate was 78.6\% (95\% CI, 62.8-88.3\%) in UP and $85.5 \%$ (95\% CI, 70.5-93.2\%) in PP. Median PFS and OS was 12.3 and 64.2 months in UP, 26.2 months and not reached in PP, respectively. Grade 3/4 febrile neutropenia was more frequent in the UP group (14.0\% vs. $2.0 \%)$.

Conclusions: Both UP and PP with IFRT achieved the expected 2-year OS. PP engendered more favorable OS and PFS compared to UP in terms. 
Keywords: Concurrent chemoradiotherapy (CCRT); non-squamous non-small cell lung cancer (NSCLC); uracil/ tegafur (UFT); pemetrexed; involved-field radiotherapy (IFRT)

Submitted Jun 09, 2020. Accepted for publication Dec 14, 2020.

doi: $10.21037 /$ tlcr-20-721

View this article at: http://dx.doi.org/10.21037/tlcr-20-721

\section{Introduction}

Inoperable stage IIIA and IIIB diseases account for 20-30\% of non-small-cell lung cancer (NSCLC) patients (1). A combination of thoracic radiotherapy (TRT) and chemotherapy is regarded as the standard treatment for inoperable stage IIIA and IIIB diseases. Although the intent of treatment is curative, most patients rapidly progress, and their prognosis is poor, with 5 -year overall survival (OS) rate in the $15-25 \%$ range (2). Several randomized trials have demonstrated cisplatin-based chemotherapy with concurrent TRT (c-TRT) is superior to that with sequential TRT in terms of response and survival. Although there are several candidate regimens that could be applied for concurrent chemoradiotherapy (CCRT), the best radiotherapy-containing combination regimen remains unclear. Though patients with locally advanced-NSCLC have been treated with a regimen of cisplatin-etoposide on the basis of evidence in the US (3), this regimen has not been approved in Japan. Docetaxel-cisplatin or vinorelbinecisplatin has been developed for chemoradiotherapy in Japan (4). A phase III study showed that cisplatin-docetaxel was superior to mitomycin-vindesine-cisplatin (MVP) in the context of chemoradiotherapy with full-dose radiation (5). Although several studies of cisplatin-vinorelbine yielded poor evidence of efficacy, this combination is frequently used in Japan when selecting a cisplatin-based regimen $(4,6)$.

We conducted a multi-institutional phase II trial of uracil-tegafur (UFT) and cisplatin (UP) with TRT for LANSCLC and compared it to vinorelbine-cisplatin (NP) with TRT (7). UFT is an antimetabolite that has been approved in Japan, and UFT monotherapy is considered as standard adjuvant treatment for Stage IA/IB/IIA lung cancer patients with primary tumors of more than $2-\mathrm{cm}$ following resection. This study showed that the UP arm and NP arm in this study met its primary endpoint of response rate. UP showed better efficacy and safety with lower hematological toxicity compared with NP (a RR of $80.0 \% / 71 \%$, MST of $26.6 / 23.9$ months, and 2-year survival rate of $54.3 \% / 48.7 \%$, respectively). Consequently, we decided to select a UP arm for the next step.

Treatment with platinum + pemetrexed (PEM) has been widely utilized for metastatic non-squamous NSCLC (8). We considered this regimen as the next candidate for chemoradiotherapy. A global phase III study comparing platinum + PEM (PP) to platinum + etoposide (PE) as an agent with radiotherapy for non-squamous NSCLC did not find that PP was superior to PE in terms of OS (9). The results of this phase III study were released after initiation of the study we describe below. We believe that additional studies including our current report are required in order to determine whether PP should be the chemoradiotherapy regimen of choice in this clinical context.

To choose a suitable phase III trial candidate for the future of LA-NSCLC treatment, we conducted a randomized phase II study comparing PP to UP with 66 Gy involved-field radiotherapy (IFRT).

We present the following article in accordance with the CONSORT reporting checklist (available at http://dx.doi. org/10.21037/tlcr-20-721).

\section{Methods}

\section{Patient eligibility}

Patients were collected by investigators in each institution belonging to North Japan Lung Cancer Study Group (NJLCG).

Eligible patients were $20-75$ years, with histologically or cytologically proven stage III non-squamous NSCLC not amenable for surgical resection, and an Eastern Cooperative Oncology Group performance status of 0 or 1 . Patients had measurable disease with RECIST criteria, or evaluable disease on computed tomography (CT) imaging and adequate organ function.

Patients were excluded if volume irradiated at $20 \mathrm{~Gy}$ (V20) cannot be reduced to less than 35\% volume of total lung. or if they had had prior systemic chemotherapy, chest radiotherapy, or surgery for NSCLC. Laboratory requirements included a leukocyte count of $4,000 / \mathrm{mm}^{3}$ or 
more, a neutrophil count of $2,000 / \mathrm{mm}^{3}$ or more, a platelet count of $100,000 / \mathrm{mm}^{3}$ or more, a hemoglobin level of $9.0 \mathrm{~g} / \mathrm{dL}$ or more, a total bilirubin level of $1.5 \mathrm{mg} / \mathrm{dL}$ or less, an AST/ALT value of twice the upper normal limit or less, a creatinine level of $1.5 \mathrm{mg} / \mathrm{dL}$ or less, a creatinine clearance of $60 \mathrm{~mL} / \mathrm{min}$ or more, and partial pressure of arterial oxygen of 70 torr or more, or $\mathrm{SpO}_{2}$ of $95 \%$ or more.

Patients were ineligible if they had concomitant malignancies, malignant pleural or pericardial effusion, or malignant ascites, interstitial pneumonitis or pulmonary fibrosis overt with chest CT, serious complications (uncontrolled diabetes mellitus, heart failure, respiratory failure, renal failure, or hepatic failure).

For staging, all patients underwent CT of the thorax and abdomen, and either brain CT or brain magnetic resonance imaging. An ${ }^{18} \mathrm{~F}$-fluorodeoxyglucose positron emission tomography (FDG-PET) was also performed on all patients.

This study was conducted in compliance with Good Clinical Practice guidelines and the Declaration of Helsinki (as revised in 2013). The study protocol was approved by the participating institution's Institutional review board (approval number 2010-016). All patients provided written informed consent before enrollment.

\section{Treatment schedules}

In this open-label study, eligible patients were stratified by age (59>/60-64/65-69/70-75), sex (female vs. male), disease stage [IIIA $v s$. IIIB (TNM $7^{\text {th }}$ edition)], and EGFR mutation status (positive/wild type/unknown) and were randomly assigned (1:1) to one of two arms by NJLCG staffs with dynamic allocation. The PP arm received pemetrexed $500 \mathrm{mg} / \mathrm{m}^{2}$ intravenously (IV) plus cisplatin $75 \mathrm{mg} / \mathrm{m}^{2} \mathrm{IV}$ every 3 weeks for three cycles with c-TRT. PP arm received daily folic acid, beginning at least 1 week before the first dose of pemetrexed, and continuing daily until 3 weeks after the last dose of pemetrexed. Intramuscular vitamin B12 was begun at least 1 week before the first dose of pemetrexed and continued every 9 weeks until 3 weeks after the last dose of pemetrexed. UP arm received oral UFT $400 \mathrm{mg} / \mathrm{m}^{2} /$ day twice daily from days 1 to 14 and cisplatin $80 \mathrm{mg} / \mathrm{m}^{2}$ IV on day 8 every 3 weeks for three cycles and c-TRT. Consolidation was not allowed in either arm. Chemotherapy was terminated after 3 cycles of chemotherapy with radiotherapy (Figure $\mathrm{S} 1$ ).

\section{Radiotherapy}

All patients were treated using a linear accelerator photon beam of $4 \mathrm{MV}$ or more from day 1 . The primary tumor and involved nodal disease received 66 Gy in 33 fractions. In this protocol, a four-dimensional (D) treatment planning system was allowed. To determine the target volume, we used FDG-PET with all patients and delineated the primary tumor and lymph nodes that were more than $1 \mathrm{~cm}$ in the short axis or were PET-positive as the gross tumor volume (GTV) or internal target volumes (ITV).

A clinical target volume (CTV) margin of $5-10 \mathrm{~mm}$ was usually added to the GTV according to the pathology. A planning target volume margin of $10 \mathrm{~mm}$ was usually added, which included the reproducibility of respiratory motion and setup error to CTV. Elective nodal irradiation was prohibited in principal to overcome dose limitation by pulmonary and esophageal toxicities.

Involved field irradiation was chosen in this study, and it is recommended that $66 \mathrm{~Gy}$ is administered in a common irradiation field using this approach. However, irradiation field reduction was permitted at the radiologist's discretion. For example, when the target lymph node is distant from the primary lesion, split-field irradiation was permitted. The maximum dose that can be received by the spinal cord was 45 Gy or less. It was also recommended that V20 should be $35 \%$ or less.

\section{Treatment modifications}

The administration of cisplatin was suspended on either arm under the following conditions: if there was a decrease in the neutrophil count to under $1,500 / \mathrm{mm}^{3}$, or the platelet count to less than $100,000 / \mathrm{mm}^{3}$, if creatinine concentration was more than $1.5 \mathrm{mg} / \mathrm{dL}$, or if grade 2 or more nonhematological toxicities (with the exception of hyponatremia) were observed. Cisplatin treatment could not be restarted until resolution of toxicity to grade 0 or 1. In the UP arm, in the event of grade 4 hematologic toxicity or grade 3 non-hematologic toxicity except for alopecia, anorexia, or malaise, the administration of UFT was stopped and then reduced in subsequent cycles from $600 \mathrm{mg}$ or 500 to $400 \mathrm{mg}$ or $300 \mathrm{mg}$, respectively. UFT was reduced whenever grade 2 diarrhea or stomatitis occurred. In the PP arm, pemetrexed was stopped and then reduced from 500 to 400 to $350 \mathrm{mg} / \mathrm{m}^{2}$, respectively if any grade 4 hematologic toxicity, or creatinine concentration more than 
$1.5 \mathrm{mg} / \mathrm{dL}$, or grade 3 non-hematologic toxicity except for hyponatremia, were observed. TRT was withheld on either arm in cases of any grade 4 hematologic toxicity, grade 3 esophagitis or dermatitis, grade 1 fever, or any sign of pneumonitis. Patients who did not receive the next cycle within 7 days, discontinued the study treatment.

\section{Treatment assessment and toxicity evaluation}

Baseline evaluations included medical history, a physical examination, electrocardiogram, tumor status, ECOG performance status, and clinical laboratory tests. Blood cell counts and biochemistry tests were performed once a week during the treatment period. Thoracic CT was performed every 4 weeks during and after the treatment period until progressive disease was recognized.

During the extramural review, tumor response was evaluated according to criteria in RECIST version 1.1. Progression-free survival (PFS) was defined as the period from the date of randomization to the date when disease progression was first observed or death occurred. These events were confirmed by several experienced physicians in the periodic extramural review. OS was defined as the period between randomization and death from any cause. Toxicities were assessed on the basis of Common Terminology Criteria for Adverse Events (CTCAE) version 4.0.

\section{Statistical analysis}

The primary end point was 2-year OS, and secondary end points including objective response rate (ORR), PFS, OS, toxicity profile. Assuming a 2-year OS of $55 \%$ in eligible patients would indicate potential usefulness, while a 2 -year OS of $35 \%$ would be the lower limit of interest, with alpha $=0.05$ and beta $=0.20$, the estimated accrual was 39 patients in each arm. Allowing for a certain number of dropouts, the accrual goal was determined to be 42 patients in each arm.

In this study, 3 cycles of chemotherapy and 66 Gy of radiation therapy were combined. Therefore, when 10 patients were registered, the safety assessment committee evaluated risks and determined whether it was safe for each patient to continue. The analysis of the primary end point was performed 2 years after the last patient was enrolled in this study.

Fisher's exact test was used to estimate the correlation among different variables between arms. Survival estimation was performed according to the Kaplan-Meier method and evaluated with the log-rank test. This trial is registered with the University Hospital Medical Information Network Clinical Trials Registry (UMIN000003948).

\section{Results}

\section{Patient characteristics}

Between November 2010 and June 2017, 86 patients were enrolled from 11 institutions and were allocated to the UP arm $(n=43)$ and PP arm $(n=43)$. Of the 86 patients enrolled, 1 patient was excluded from final analysis due to ineligibility (V20 35\% <). Therefore, 85 patients (UP arm, n=43 and PP arm, $\mathrm{n}=42$ ) were evaluable for efficacy and safety (Figure 1). Baseline patient and disease characteristics were well balanced in terms of age, gender, stage, and EGFR mutation (Table 1); these characteristics were used as stratification factors. Regarding performance state, the PP arm was slightly biased toward PS1 when compared to the UP arm.

\section{Treatment administered}

As shown in Table 2, the median number of treatment cycles was 3 (range, 1-3) in both arms. In the UP and PP arms, $74.0 \%$ and $85.0 \%$ of patients underwent the three cycles of chemotherapy with radiotherapy, respectively. The slightly lower completion rates of the three cycles of the UP arm may be related to myelosuppression and the schedule, in which UFT started on day 1 and cisplatin chemotherapy started on day 8. TRT at 66 Gy was completed in 39 of the 43 patients (91\%) in the UP arm and 38 of 42 patients (90\%) in the $\mathrm{PP}$ arm.

\section{Efficacy}

The ORR was $76.7 \%$ (95\% CI, 61.0-87.7\%) versus $81.0 \%$ (95\% CI, 65.4-90.9\%), and the disease control rates (DCR) were $90.7 \%$ (95\% CI, $76.9-97.0 \%$ ) versus $100 \%$ (95\% CI, 89.6-100\%), for the UP arm versus PP arm, respectively (Table 3). The response rate of the PP group was better than that of the UP group, but the difference between the two groups was not statistically significant $(\mathrm{P}=0.7916)$.

The Kaplan-Meier curves of OS and PFS are shown in Figure 2. Most of the patients were observed for more than 2 years and median follow-up time for the censored patients was 54 months; survival events occurred in 31 patients. The median survival time was 64.2 months for the UP arm and not reached for the PP arm. However, there was no statistically significant difference between the two arms in 


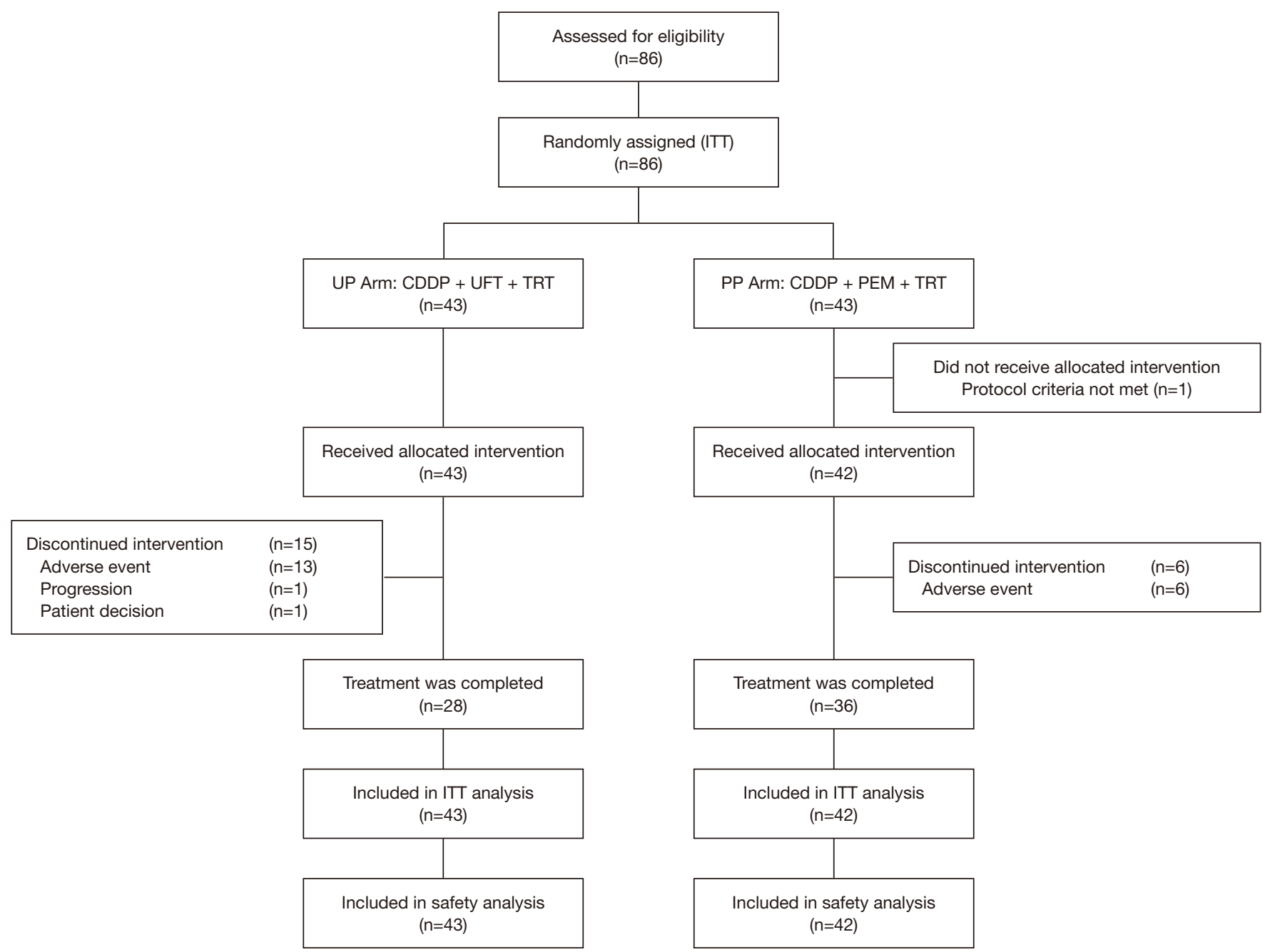

Figure 1 CONSORT diagram. 86 patients were enrolled from 11 institutions. Finally 85 patients were evaluable for efficacy and safety.

terms of OS (HR $=0.612 ; 95 \% \mathrm{CI}, 0.30-1.27 ; \mathrm{P}=0.18)$. The median PFS was 12.3 months (95\% CI, 9.2-19.2 months) for the UP arm and 26.2 months (95\% CI, 17.6-69.3 months) for the PP arm. PFS in the PP arm was more than twice as long as in the UP arm, but there was also no statistically significant difference in PFS between the arms ( $\mathrm{HR}=0.605$; 95\% CI, 0.36-1.02; $\mathrm{P}=0.06)$. The 2 -year OS rates were $78.6 \%$ (95\% CI, $62.8-88.3 \%$ ) and $85.5 \%$ (95\% CI, 70.593.2\%) with the UP arm and the PP arm, respectively. The lower limit of the CI for 2-year OS in both arms exceeded the threshold of $50 \%$. Subset analyses showed that OS was not significantly different when various factors were taken into consideration (Figure 3).

Disease recurrences were found in 31 patients in the UP arm and 28 patients in the PP arm. In-field relapse was observed in 4 patients (12.9\%) in the UP arm and 2 patients $(8.7 \%)$ in the PP arm. Distant metastases were observed in 27 patients $(87.1 \%)$ in the UP arm and 21 patients $(91.3 \%)$ in the PP arm.

\section{Safety}

Treatment-emergent AEs possibly related to study treatment are listed in Table 4. Grade $3 / 4$ neutropenia occurred in $34.9 \%$ and $31.0 \%$ of patients in UP and PP arms, respectively.

Grade 3/4 febrile neutropenia was more frequent in the UP arm than in the PP arm (14.0\% and 2.0\%, respectively). Grade $3 / 4$ pneumonitis was present in $7.0 \%$ of the UP arm and $4.8 \%$ of the PP arm. Grade 3 or higher anorexia 
Table 1 Patient characteristics

\begin{tabular}{|c|c|c|}
\hline Characteristics & UP arm, $n=43(\%)$ & PP arm, $n=42(\%)$ \\
\hline \multicolumn{3}{|l|}{ Age } \\
\hline$\leq 59$ & 17 (39.5) & $15(35.7)$ \\
\hline $60-64$ & $10(23.3)$ & $12(28.6)$ \\
\hline $65-69$ & 7 (16.3) & $6(14.3)$ \\
\hline $70-75$ & $9(20.9)$ & $9(21.4)$ \\
\hline Median & 62.0 & 62.5 \\
\hline \multicolumn{3}{|l|}{ Gender } \\
\hline Male & $32(74.4)$ & $34(81.0)$ \\
\hline Female & $11(25.6)$ & $8(19.0)$ \\
\hline \multicolumn{3}{|l|}{ PS (ECOG) } \\
\hline 0 & $29(67.4)$ & $33(78.6)$ \\
\hline 1 & $14(32.6)$ & $9(21.4)$ \\
\hline \multicolumn{3}{|l|}{ Stage } \\
\hline IIIA & $23(53.5)$ & $24(57.1)$ \\
\hline IIIB & $20(46.5)$ & $18(42.9)$ \\
\hline \multicolumn{3}{|l|}{ Smoking history } \\
\hline Current/former & $39(90.7)$ & $36(85.7)$ \\
\hline Never & $4(9.3)$ & $6(14.3)$ \\
\hline \multicolumn{3}{|l|}{ EGFR mutation } \\
\hline Positive & 7 (16.3) & $9(21.4)$ \\
\hline Wild type & $32(74.4)$ & $32(76.2)$ \\
\hline Unknown & $4(9.3)$ & $1(2.4)$ \\
\hline
\end{tabular}

UP, UFT and cisplatin; PP, pemetrexed and cisplatin; PS, performance status; ECOG, Eastern Cooperative Oncology Group; EGFR, epidermal growth factor receptor.

and diarrhea were more common in the UP arm. No one had skin disorders of grade 3 or worse in either arm. No treatment-related deaths occurred during the study period.

\section{Discussion}

In this study, both groups met the primary endpoints of 2-year OS, with $78.6 \%$ (95\% CI, 62.8-88.3\%) in the UP group and $85.5 \%$ (95\% CI, 70.5-93.2\%) in the PP group. The median PFS was significantly longer in the PP group than in the UP group. The improved PFS did not translate to a significant effect on OS. These results are consistent with a previously reported association between PFS and OS
Table 2 Treatment delivery

\begin{tabular}{lcc}
\hline Characteristics & UP arm, $\mathrm{n}=43(\%)$ & PP arm, $\mathrm{n}=42(\%)$ \\
\hline Cycle number & $6(14.0)$ & $4(9.5)$ \\
1 & $5(11.6)$ & $2(4.8)$ \\
2 & $32(74.4)$ & $36(85.7)$ \\
3 & 3 & 3 \\
Median & & \\
Radiation dose (Gy) & $39(90.7)$ & $38(90.5)$ \\
66 & $1(2.3)$ & $0(0.0)$ \\
$60-65$ & $1(2.3)$ & $0(0.0)$ \\
$50-59$ & $0(0.0)$ & $1(2.4)$ \\
$40-49$ & $2(4.7)$ & $3(7.1)$ \\
$<40$ & 66 & 66 \\
Median & UP, UFT and cisplatin; PP, pemetrexed and cisplatin.
\end{tabular}

in lung cancer patients, where long post-progression survival counteracts the effects of first-line treatment (10-12).

Our study revealed a significant efficacy of PFS and OS in both arms. We suggest that this efficacy can be attributed to the design of radiotherapy schedule. Although the standard radiation dose was $60 \mathrm{~Gy}$ in practice and in most trials, we selected a $66-G y$ dose in this trial $(5,13)$. In the RTOG 0617 trial, the efficacy of radiotherapy at either 60 or 74 Gy with chemotherapy \pm cetuximab was evaluated (14). The results showed that 74 Gy treatment was associated with poorer survival when compared with $60 \mathrm{~Gy}$; the toxicities were not significantly different in either radiation dose cohort, regardless of cetuximab treatment. There have been no studies comparing 66 and 60 Gy, and we selected 66 Gy as the feasible high dose radiotherapy. Another difference between our study and those carried out previously is the method by which radiotherapy was administered. In this study, IFRT was performed instead of conventional radiotherapy that includes elective nodal irradiation. Involved-field RT can decrease the number of radiation fields required while providing an increased radiation dose. Previous studies did not observe statistically significant differences in efficacy in patients treated with IF-RT versus those treated with conventional RT $(15,16)$. Next, we excluded consolidation of chemotherapy from the chemoradiotherapy schedule. During the development of this protocol, severe radiation pneumonitis was observed during 
Table 3 Responses in treated patients

\begin{tabular}{lcc}
\hline & UP arm, $\mathrm{n}=43(\%)$ & $\mathrm{PP}$ arm, $\mathrm{n}=42(\%)$ \\
\hline CR & $3(7.0)$ & $1(2.4)$ \\
PR & $30(69.8)$ & $33(78.6)$ \\
SD & $6(14.0)$ & $8(19.0)$ \\
PD & $2(4.7)$ & $0(0.0)$ \\
Not evaluable & $2(4.7)$ & $0(0.0)$ \\
ORR, $[95 \% \mathrm{Cl}]$ & $33(76.7),[61.0-87.7]$ & $34(81.0),[65.4-90.9]$ \\
DCR, $[95 \% \mathrm{Cl}]$ & $39(90.7),[76.9-97.0]$ & $42(100),[89.6-100]$ \\
\hline
\end{tabular}

UP, UFT and cisplatin; PP, pemetrexed and cisplatin; CR, complete response; PR, partial response; SD, stable disease; PD, progressive disease; ORR, overall response rate; $\mathrm{DCR}$, disease control rate; $\mathrm{Cl}$, Confidence interval.
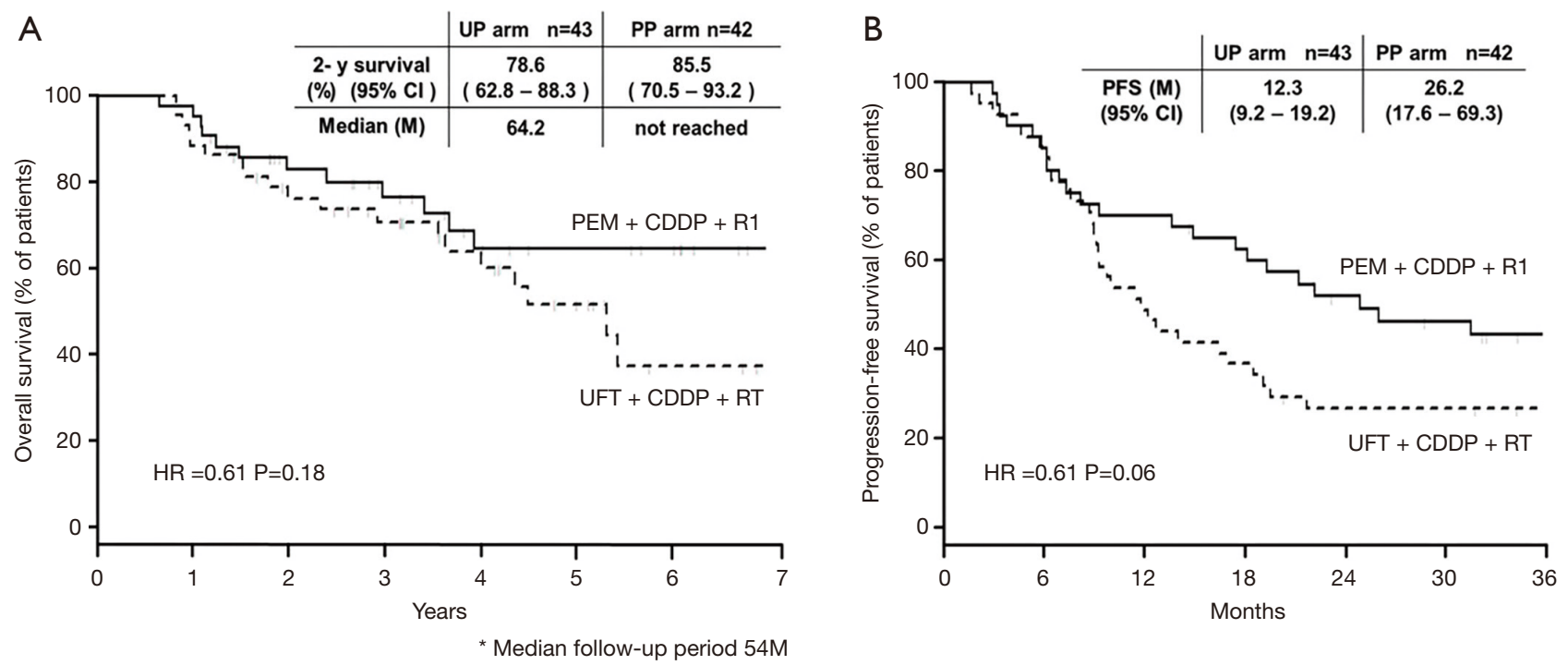

Figure 2 Kaplan-Meier curves. (A) Overall survival and (B) progression-free survival for the randomly assigned treatment arms.

the consolidation phase in a phase 1 study in Japan (17). Generally, the evidence that consolidation after chemoradiotherapy is efficacious and safe is poor according to the JCO guidelines and a pooled analysis (18).

The use of consolidation chemotherapy remains controversial. After chemoradiation, consolidation chemotherapy did not improve OS and is not currently recommended. A pooled analysis of forty-two studies comparing consolidation chemotherapy after CCRT with best supportive care showed no difference in median OS $(\mathrm{P}=0.4)$. Consequently, in this study three cycles of chemotherapy were administered during 66-Gy radiation without consolidation chemotherapy.

Our current study demonstrated that, when compared to cisplatin plus UFT, a regimen of cisplatin plus pemetrexed achieved superior PFS and improved OS, although both PFS and OS was not statistically significant. Our study revealed a longer PFS and OS than those reported by PROCLAIM phase III study comparing cisplatin plus pemetrexed to cisplatin plus etoposide (9). PROCLAIM study could not achieve the primary endpoint to validate OS benefit. (2 year-OS: $52 \%$, PFS: 17.4). One of the differences between this study and the PROCLAIM study were whether consolidation was a feature. We note that various consolidation treatments were accepted in the PROCLAIM study, but not accepted in our study. Another difference is race. Specifically, although the $23 \%$ of patients in the PROCLAIM study included East Asians (but not 
A

$\begin{array}{lcc}\text { Subgroup } & \text { UP } & \text { PP } \\ \text { Overall } & 43 & 42 \\ \text { Age (years) } & & \\ -59 & 17 & 15 \\ 60-64 & 10 & 12 \\ 65-69 & 7 & 6 \\ 70- & 9 & 9 \\ \text { Sex } & & \\ \text { Female } & 11 & 8 \\ \text { Male } & 32 & 34 \\ \text { EGFR mutation } & & \\ \text { Positive } & 7 & 9 \\ \text { Wild } & 32 & 32 \\ \text { Clinical Stage } & & \\ \text { IIIA } & 23 & 24 \\ \text { IIIB } & 20 & 18\end{array}$

\section{B}

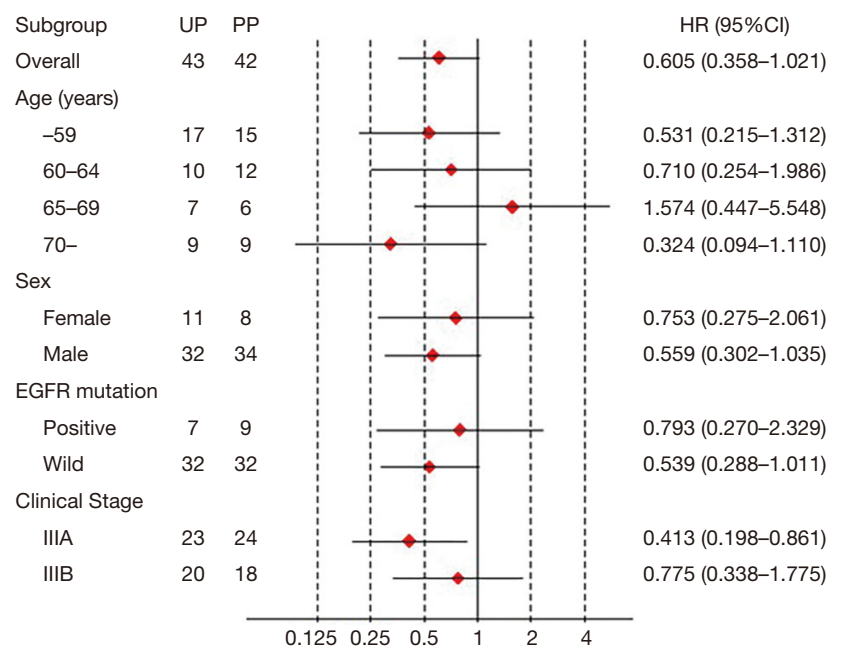

Figure 3 Subgroup analysis. Overall survival (A) and progression-free survival (B) hazard ratio in subgroups according to baseline characteristics.

Table 4 Toxicity profiles

\begin{tabular}{|c|c|c|c|c|}
\hline & \multicolumn{2}{|c|}{ UP arm, n=43 (\%) } & \multicolumn{2}{|c|}{ PP arm, n=42 (\%) } \\
\hline & All grades & Grade 3 or 4 & All grades & Grade 3 or 4 \\
\hline Leukopenia & $40(93.0)$ & $20(46.5)$ & $29(69.0)$ & $15(35.7)$ \\
\hline Neutropenia & $35(81.4)$ & 15 (34.9) & 27 (64.3) & $13(31.0)$ \\
\hline Anemia & $27(62.8))$ & $3(7.0)$ & $22(52.4)$ & $0(0.0)$ \\
\hline Thrombocytopenia & $19(44.2)$ & $4(9.3)$ & $14(33.3)$ & $2(4.8)$ \\
\hline Febrile neutropenia & $6(14.0)$ & $6(14.0)$ & $1(2.4)$ & $1(2.4)$ \\
\hline Anorexia & $25(58.1)$ & $8(18.6)$ & $23(54.8)$ & $1(2.4)$ \\
\hline Nausea/Vomiting & $3(7.0)$ & $0(0.0)$ & $2(4.8)$ & $0(0.0)$ \\
\hline Diarrhea & $9(20.9)$ & $5(11.6)$ & $1(2.4)$ & $0(0.0)$ \\
\hline Constipation & $11(25.6)$ & $0(0.0)$ & $15(35.7)$ & $0(0.0)$ \\
\hline Infection & $2(4.7)$ & $2(4.7)$ & $5(11.9)$ & $3(7.1)$ \\
\hline AST/ALT increased & $17(39.5)$ & $2(4.7)$ & $5(11.9)$ & $0(0.0)$ \\
\hline Pneumonitis & $33(76.7)$ & $3(7.0)$ & $36(85.7)$ & $2(4.8)$ \\
\hline Esophagitis & $22(51.2)$ & $2(4.7)$ & $16(38.1)$ & $2(4.8$ \\
\hline Dermatitis & $12(27.9)$ & $0(0.0)$ & $20(47.6)$ & $1(2.4)$ \\
\hline
\end{tabular}

UP, UFT and cisplatin; PP, pemetrexed and cisplatin; AST, aspartate aminotransferase; ALT, alanine aminotransferase. 
the Japanese), our current study was entirely composed of Japanese individuals. The Japanese studies of lung cancer have often revealed longer PFS and OS compared to studies of Western populations. This difference can be explained by both racial differences and a Japanese insurance system that supports the provision of the most appropriate line of cancer treatment according to the individual's clinical characteristics. Actually, $87 \%$ of patients in this study received second-line or later therapies after conclusion of the study treatment, whereas this figure was only $42.5 \%$ in the PROCLAIM study. Other differences among two studies are rate of PET test. Approximately $80 \%$ of patients underwent PET in the PROCLAIM study, but in our study all cases were examined using PET, which helps with correct staging $(19,20)$. The staging may therefore have been more accurate in this study.

In terms of toxicity, more hematological toxicity was observed in the UFT group than in the PEM group; this was a major reason for failure to complete 3 cycles in the UFT group. Diarrhea was more common in the UFT group and skin disorders were more common in the PEM group. Although pneumonitis was a concern in the PEM group before initiation of the study, the percentage of grade 3 or higher pneumonitis cases was $7.0 \%$ in the UFT group and $4.8 \%$ in the PEM group. Taken together, toxicities in both regimens were expected and manageable.

Recently, the PACIFIC trial, a phase III study of chemoradiation with consolidation using durvalumab inhibiting PD-L1 compared to without consolidation in patients with stage III unresectable NSCLC, demonstrated statistically significant increases in OS and PFS $(21,22)$. Consolidation with durvalumab following CCRT became a new standard of care for patients with locally advanced NSCLC. Important things in this situation are to select efficacious and safety chemoradiation methods to connect successfully to consolidation with durvalumab. This CCRT of 66 Gy IF-RT with cisplatin plus pemetrexed may thus have therapeutic potential as an appropriate CCRT followed by durvalumab for inoperable stage III nonsquamous NSCLC.

This study has several limitations. First, the study is a phase II randomized trial to select candidates for a phase III trial and thus has no confirmatory meaning. Second, the study required nine years of recruitment and observation. During this period, the parameters related to some standard treatment regimens and TNM classification have changed. However, studies with a small number of locally advanced patients compared to studies with metastatic patients are always faced with challenges in recruitment.

In conclusion, PP was safer and more efficacious than UP. Thus, in a future phase III study, PP should be considered as the experimental arm for comparison to the standard regimen with c-TRT for non-squamous NSCLC.

\section{Acknowledgments}

We thank all patients and their families, as well as all investigators for their support in this study. We presented the outline of this study at the 2019 ASCO Annual Meeting. Funding: None.

\section{Footnote}

Reporting Checklist: The authors have completed the CONSORT reporting checklist. Available at http://dx.doi. org/10.21037/tlcr-20-721

Data Sharing Statement: Available at http://dx.doi. org/10.21037/tlcr-20-721

Conflicts of Interest: All authors have completed the ICMJE uniform disclosure form (available at http://dx.doi. org/10.21037/tlcr-20-721). YT reports personal fees from AstraZeneca, Bristol-Myers Squibb, MSD, Ono Pharmaceutical, outside the submitted work. Atsushi Nakamura reports personal fees from MSD, AstraZeneca, Boehringer Ingelheim Japan, outside the submitted work. EM reports grants and personal fees from Chugai pharmaceutical co ltd, Ono pharmaceutical co., ltd., Boehringer Ingelheim, Eli Lilly Japan, and personal fees from AstraZeneca, Taiho pharma, Kyowa kirin, Daiichi sankyo, MSD, Bristol-Meyers Squibb, Novartis, Merck Bio, outside the submitted work. HM reports personal fees from Ono Pharmaceutical Co., Ltd., Nippon Boehringer Ingelheim Co., Ltd., Eli Lilly Japan, Chugai Pharmaceutical Co., Ltd., outside the submitted work. TF reports grants from Ono Pharmaceutical Co., Bristol Myers Squibb, AstraZeneca, MSD, outside the submitted work. AI reports personal fees from Eli Lilly Japan, outside the submitted work. Shunichi Sugawara reports personal fees from Eli Lilly Japan, Taiho Pharmaceutical, MSD, AstraZeneca, Bristol-Myers Squibb, Ono Pharmaceutical, Chugai Pharma, Nippon Boehringer Ingelheim, Pfizer, Novartis, Kyowa Kirin, Yakult Honsha, outside the submitted work. MM reports personal fees from Eli Lilly Japan, grants from Taiho, outside the submitted work. The other authors have 
no conflicts of interest to declare.

Ethical Statement: The authors are accountable for all aspects of the work in ensuring that questions related to the accuracy or integrity of any part of the work are appropriately investigated and resolved. This study was conducted in compliance with Good Clinical Practice guidelines and the Declaration of Helsinki (as revised in 2013). The study protocol was approved by the participating institution's Institutional review board (approval number 2010-016). All patients provided written informed consent before enrollment.

Open Access Statement: This is an Open Access article distributed in accordance with the Creative Commons Attribution-NonCommercial-NoDerivs 4.0 International License (CC BY-NC-ND 4.0), which permits the noncommercial replication and distribution of the article with the strict proviso that no changes or edits are made and the original work is properly cited (including links to both the formal publication through the relevant DOI and the license). See: https://creativecommons.org/licenses/by-nc-nd/4.0/.

\section{References}

1. Morgensztern D, Ng SH, Gao F, et al. Trends in Stage Distribution for Patients with Non-Small Cell Lung Cancer: A National Cancer Database Survey. J Thorac Oncol 2010;5:29-33.

2. Liang J, Bi N, Wu S, et al. Etoposide and cisplatin versus paclitaxel and carboplatin with concurrent thoracic radiotherapy in unresectable stage III non-small cell lung cancer: a multicenter randomized phase III trial. Ann Oncol 2017;28:777-83.

3. Bezjak A, Temin S, Franklin G, et al. Definitive and Adjuvant Radiotherapy in Locally Advanced Non-SmallCell Lung Cancer: American Society of Clinical Oncology Clinical Practice Guideline Endorsement of the American Society for Radiation Oncology Evidence-Based Clinical Practice Guideline. J Clin Oncol 2015;33:2100-5.

4. Naito Y, Kubota K, Nihei K, et al. Concurrent Chemoradiotherapy With Cisplatin and Vinorelbine for Stage III Non-Small Cell Lung Cancer. J Thorac Oncol 2008;3:617-22.

5. Segawa Y, Kiura K, Takigawa N, et al. Phase III Trial Comparing Docetaxel and Cisplatin Combination Chemotherapy With Mitomycin, Vindesine, and Cisplatin Combination Chemotherapy With Concurrent Thoracic
Radiotherapy in Locally Advanced Non-Small-Cell Lung Cancer: OLCSG 0007. J Clin Oncol 2010;28:3299-306.

6. Sekine I, Noda K, Oshita F, et al. Phase I study of cisplatin and vinorelbine for unresectable stage III non-small-cell lung cancer. Cancer Sci 2004;95: 691-5.

7. Sugawara S, Maemondo M, Tachihara M, et al. Randomized phase II trial of uracil/tegafur and cisplatin versus vinorelbine and cisplatin with concurrent thoracic radiotherapy for locally advanced unresectable stage III non-small-cell lung cancer: NJLCG 0601. Lung Cancer 2013;81:91-6.

8. Scagliotti G, Brodowicz T, Shepherd FA, et al. Treatmentby-histology Interaction Analyses in Three Phase III Trials Show Superiority of Pemetrexed in Nonsquamous NonSmall Cell Lung Cancer. J Thorac Oncol 2011;6:64-70.

9. Senan S, Brade A, Wang LH, et al. PROCLAIM: Randomized Phase III Trial of Pemetrexed-Cisplatin or Etoposide-Cisplatin Plus Thoracic Radiation Therapy Followed by Consolidation Chemotherapy in Locally Advanced Nonsquamous Non-Small-Cell Lung Cancer. J Clin Oncol 2016;34:953-62.

10. Hayashi H, Okamoto I, Taguri M, et al. Postprogression Survival in Patients With Advanced Non-Small-Cell Lung Cancer Who Receive Second-Line or Third-Line Chemotherapy. Clin Lung Cancer 2013;14:261-6.

11. Hotta K, Kiura K, Fujiwara Y, et al. Role of survival postprogression in phase III trials of systemic chemotherapy in advanced non-small-cell lung cancer: A systematic review. PLOS One 2011;6:e26646.

12. Hayashi H, Okamoto I, Morita S, et al. Postprogression survival for first-line chemotherapy of patients with advanced non-small-cell lung cancer. Ann Oncol 2012;23:1537-41.

13. Yamamoto N, Nakagawa K, Nishimura Y, et al. Phase III study comparing second-and third-generation regimen with concurrent thoracic radiotherapy in patients with unresectable stage III non-small-cell lung cancer. West Japan Thoracic Oncology Group WJTOG0510. J Clin Oncol 2010;28:3739-45.

14. Bradley JD, Paulus R, Komaki R, et al. Standard-dose versus high-dose conformal radiotherapy with concurrent and consolidation carboplatin plus paclitaxel with or without cetuximab for patients with stage IIIA or IIIB non-smallcell lung cancer (RTOG 0617): A randomised, two-by-two factorial phase 3 study. Lancet Oncol 2015;16:187-99.

15. Ruijian Li, Liang Yu, Sixiang Lin, et al. Involved field radiotherapy (IFRT) versus elective nodal irradiation (ENI) for locally advanced non-small cell lung cancer: a 
meta-analysis of incidence of elective nodal failure (ENF). Radiat Oncol 2016;11:124.

16. Fernandes AT, Shen J, Finlay J, et al. Elective nodal irradiation (ENI) vs. involved field radiotherapy (IFRT) for locally advanced non-small cell lung cancer (NSCLC): a comparative analysis of toxicities and clinical outcomes. Radiother Oncol 2010;95:178-84.

17. Niho S, Kubota K, Nihei K, et al. Dose-escalation study of thoracic radiotherapy in combination with pemetrexed plus cisplatin followed by pemetrexed consolidation therapy in japanese patients with locally advanced nonsquamous nonsmall-cell lung cancer. Clin Lung Cancer 2013;14:62-9.

18. Tsujino K, Kurata T, Yamamoto S, et al. Is Consolidation Chemotherapy after Concurrent Chemo-Radiotherapy Beneficial for Patients with Locally Advanced Non-SmallCell Lung Cancer? A Pooled Analysis of the Literature. J
Thorac Oncol 2013;8:1181-9.

19. MacManus MP, Hicks RJ, Matthew JP, et al. High Rate of Detection of Unsuspected Distant Metastases by Pet in Apparent Stage III Non-Small-Cell Lung Cancer: Implications for Radical Radiation Therapy. Int J Radiat Oncol Biol Phys 2001;50:287-93.

20. Ung YC, Bezjak A, Coakley N, et al. Positron Emission Tomography with 18Fluorodeoxyglucose in Radiation Treatment Planning for Non-small Cell Lung Cancer: A Systematic Review. J Thorac Oncol 2011;6:86-97.

21. Antonia SJ, Villegas A, Daniel D, et al. Overall Survival with Durvalumab after Chemoradiotherapy in Stage III NSCLC. N Engl J Med 2018;379:2342-50.

22. Antonia SJ, Villegas A, Daniel D, et al. Durvalumab after Chemoradiotherapy in Stage III Non-Small-Cell Lung Cancer. N Engl J Med 2017;377:1919-29.
Cite this article as: Watanabe $\mathrm{K}$, Toi Y, Nakamura A, Chiba R, Akiyama M, Sakakibara-Konishi J, Tanaka H, Yoshimura N, Miyauchi E, Nakagawa T, Igusa R, Minemura H, Mori Y, Fujimoto K, Matsushita H, Takahashi F, Fukuhara T, Inoue A, Sugawara S, Maemondo M; North Japan Lung Cancer Study Group, Sendai, Japan. Randomized phase II trial of uracil/tegafur and cisplatin versus pemetrexed and cisplatin with concurrent thoracic radiotherapy for locally advanced unresectable stage III non-squamous non-small cell lung cancer: NJLCG1001. Transl Lung Cancer Res 2021;10(2):712-722. doi: 10.21037/tlcr-20-721 


\section{Supplementary}

Key eligibility criteria

Patients with unresectable, stage IIIA or IIIB non-squamous NSCLC

ECOG PS 0 or 1

Age $20-75$ years

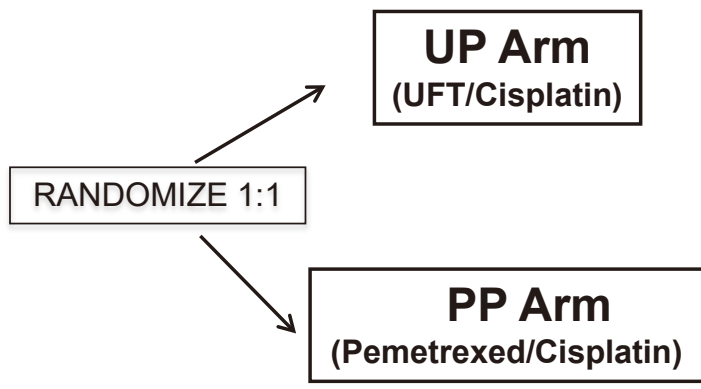

UFT $400 \mathrm{mg} / \mathrm{m}^{2}$, day $1-14$

CDDP $80 \mathrm{mg} / \mathrm{m}^{2}$, day 8

q3w $\times 3$ cycles

IFRT 2Gy x 5 days/week, day 1-45, 66Gy

PEM $500 \mathrm{mg} / \mathrm{m}^{2}$, day 1

CDDP $75 \mathrm{mg} / \mathrm{m}^{2}$, day 1

q3w $\times 3$ cycles

IFRT 2Gy x 5 days/week, day for LA-NSCLC

$\checkmark$ Consolidation

Consolidation is not allowed.

Chemotherapy is terminated after 3 cycle.

\section{$\checkmark \quad$ Stratified factor}

Age $\quad 59 \geq / 60 \sim 64 / 65 \sim 69 / 70 \sim 75$

Gender Male/Female

Stage $\quad$ IIIA/IIIB (TNM $7^{\text {th }}$ edition)

EGFR mt status positive/wild/unknown $\checkmark \quad$ Radiotherapy

Involved-field radiotherapy (IFRT) was administered from day 1

Total dose was 66Gy in 33 fractions.

\section{$\checkmark \quad$ Primary endpoint}

2-year overall survival

$\checkmark \quad$ Secondary endpoint

ORR, PFS, OS, Toxicity profile

Figure S1 Treatment schedule of each arm. This figure shows key eligibility criteria and dose of each treatment. 